This item was submitted to Loughborough's Research Repository by the author.

Items in Figshare are protected by copyright, with all rights reserved, unless otherwise indicated.

\title{
Matrix equations and Hilbert's tenth problem
}

PLEASE CITE THE PUBLISHED VERSION

http://www.worldscientific.com/doi/pdf/10.1142/S0218196708004925

PUBLISHER

(C) World Scientific Publishing

VERSION

AM (Accepted Manuscript)

LICENCE

CC BY-NC-ND 4.0

REPOSITORY RECORD

Bell, Paul C., Vesa Halava, Tero Harju, Juhani Karhumaki, and Igor Potapov. 2019. "Matrix Equations and Hilbert's Tenth Problem". figshare. https://hdl.handle.net/2134/12045. 
This item was submitted to Loughborough's Institutional Repository (https://dspace.lboro.ac.uk/) by the author and is made available under the following Creative Commons Licence conditions.

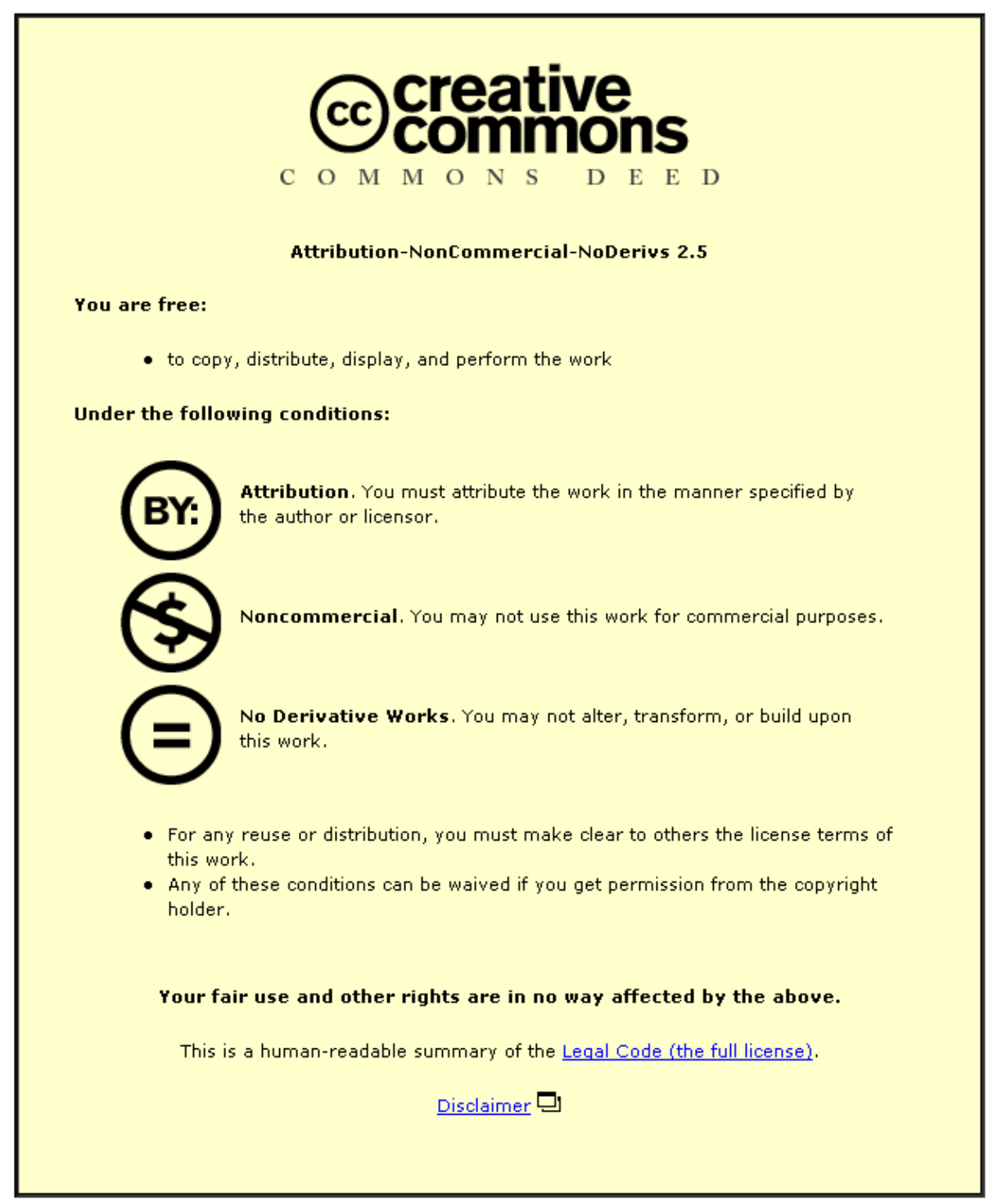

For the full text of this licence, please go to: http://creativecommons.org/licenses/by-nc-nd/2.5/ 


\title{
Matrix Equations and Hilbert's Tenth Problem
}

\author{
Paul C. Bell, Vesa Halava, Tero Harju, Juhani Karhumäki, * \\ Igor Potapov ${ }^{\dagger}$
}

\begin{abstract}
We show a reduction of Hilbert's tenth problem to the solvability of the matrix equation $X_{1}^{i_{1}} X_{2}^{i_{2}} \cdots X_{k}^{i_{k}}=Z$ over non-commuting integral matrices, where $Z$ is the zero matrix, thus proving that the solvability of the equation is undecidable. This is in contrast to the case whereby the matrix semigroup is commutative in which the solvability of the same equation was shown to be decidable in general.

The restricted problem where $k=2$ for commutative matrices is known as the "A-B-C Problem" and we show that this problem is decidable even for a pair of non-commutative matrices over an algebraic number field.
\end{abstract}

Keywords: Matrix Equations; Undecidability; Formal Power Series; Diophantine Equations.

\section{Introduction}

Matrices and matrix semigroups play a fundamental and central role in many diverse fields of mathematics and computer science. There has been a great deal of interest by researchers on computational problems for finitely generated matrix semigroups and many natural decision questions on them are in fact undecidable.

One such problem which was studied is the mortality problem. We are given a finite set of matrices, $G$, forming a semigroup $S$, and must determine whether the zero matrix (the matrix with all zero elements) is present in the semigroup. This problem was shown to be undecidable by M. Paterson in 1970 for 3-dimensional integer matrix semigroups, [17], and remains undecidable even when there are only 7 matrices in the generator of the semigroup [9].

${ }^{*}$ TUCS-Turku Centre for Computer Science, Department of Mathematics, University of Turku, FIN-20014, Turku, Finland. p.bell@lboro.ac.uk, vehalava@utu.fi, harju@utu.fi, karhumak@utu.fi.

${ }^{\dagger}$ Department of Computer Science, The University of Liverpool, Liverpool, L69-3BX, UK. potapov@liverpool.ac.uk. 
The membership problem for a scalar matrix (A matrix with a scalar $k$ on all leading diagonal elements and 0 elsewhere) was recently shown to be undecidable for 4-dimensional integral matrices, see [4]. We also mention that the freeness problem for 3-dimensional integral matrix semigroups is undecidable, see [13]. In fact, the problem remains undecidable even when the matrices are upper triangular, see [8].

What can be said of decidable cases in the area however? There are far fewer cases where decision problems are known to be decidable. It was shown that the "orbit problem" (Given a matrix $M \in \mathbb{Q}^{n \times n}$ and vectors $u, v \in \mathbb{Q}^{n}$, does there exist any $k \geq 0$ such that $M^{k} u=v$ ?) is decidable, even in polynomial time, see [12]. Furthermore, it was shown that for a semigroup generated by row-monomial rational matrices (each row of a matrix contains exactly one nonzero element), the membership is decidable for any dimension, see [14]. Some criteria for semigroup freeness in two-dimensional upper triangular matrices was also shown in [8].

Another decidable case which was shown was that for a commutative rational matrix semigroup in any dimension, where the membership problem was shown to be decidable in polynomial time, see [1]. In this problem, we are given a (finite) set of commutative matrices $G=\left\{M_{1}, M_{2}, \ldots, M_{t}\right\} \subseteq$ $\mathbb{Q}^{n \times n}$, and a fixed matrix $M$. The problem can be stated as: does there exist natural numbers $j_{1}, j_{2}, \ldots, j_{t}$ such that:

$$
M_{1}^{j_{1}} M_{2}^{j_{2}} \cdots M_{t}^{j_{t}}=M ?
$$

In this paper we shall examine a related problem where we consider the above equation for non-commutative integral matrices. We show that given the $k$ matrices $X_{1}, X_{2}, \ldots, X_{k} \subseteq \mathbb{Z}^{n \times n}$, determining whether there exists natural numbers $i_{1}, i_{2}, \ldots, i_{k}$ such that:

$$
X_{1}^{i_{1}} X_{2}^{i_{2}} \cdots X_{k}^{i_{k}}=Z,
$$

where $Z$ is the zero matrix, is undecidable. We do not use a reduction of Post's correspondence problem, as is standard for undecidability proofs, we instead use the undecidability of Hilbert's tenth problem and properties of formal power series to show the undecidability.

Given three commutative matrices $A, B, C$ the problem of determining the solvability of the equation $A^{i} B^{j}=C$ for arbitrary $i, j \geq 0$ is known as the "A-B-C Problem" and was shown to be decidable in [7] (obviously this is a sub-case of the more general decidability result of [1] but it was considered prior to this result and formulated after the results of [12]). We show that the "A-B-C Problem" is decidable even for non-commutative matrices $A, B, C$ over an algebraic number field. 


\section{Preliminaries}

\subsection{Matrices and Words}

Let $A$ be a finite set of letters called an alphabet. A word $w$ is a finite sequence of letters from $A$ and the set of all words over $A$ is denoted $A^{*}$. The empty word is denoted by $\varepsilon$. For two words $u=u_{1} u_{2} \cdots u_{i}$ and $v=$ $v_{1} v_{2} \cdots v_{j}$, where $u, v \in A^{*}$, the concatenation of $u$ and $v$ is denoted by $u \cdot v$ such that $u \cdot v=u_{1} u_{2} \cdots u_{i} v_{1} v_{2} \cdots v_{j}$. By abuse of notation, we also refer to concatenation via juxtaposition, i.e., $u \cdot v=u v$. A subset $L$ of $A^{*}$ is called a language.

As usual, for a matrix $M$, we denote by $M^{T}$ the transpose of matrix $M$. For an arbitrary semiring $K$, let vec be a function, vec : $K^{n \times n} \mapsto K^{n^{2}}$, such that vec takes an $n \times n$ matrix and creates a $n^{2}$ dimensional column vector by stacking the columns of the matrix on top of each other starting with the first, i.e., for a matrix $M \in K^{n \times n}$, then:

$\operatorname{vec}(M)=\left(M_{[1,1]}, \ldots, M_{[n, 1]}, M_{[1,2]}, \ldots, M_{[n, 2]}, \ldots, M_{[1, n]}, \ldots, M_{[n, n]}\right)^{T} \in K^{n^{2}}$

Let $A, B, C, X \in K^{n \times n}$, then it is well known that the equation $A X B=$ $C$ (for unknown $X$ ), can be rewritten:

$$
\left(B^{T} \otimes A\right) \operatorname{vec}(X)=\operatorname{vec}(C),
$$

where $\otimes$ denotes the Kronecker product, see [5].

We shall also need the mixed product property of Kronecker products, namely that for given matrices $A, B, C, D \in K^{n \times n}$ it holds that:

$$
(A \otimes B)(C \otimes D)=(A C \otimes B D) \in K^{n^{2} \times n^{2}} .
$$

\subsection{Formal Power Series}

We use the definitions and terminology as in [6]. Here, and throughout, let $K$ be a semiring and $A$ a finite alphabet generating a free monoid denoted by $A^{*}$. A formal power series, $S$, is defined to be a function:

$$
S: A^{*} \mapsto K
$$

and the image of a word $w \in A^{*}$ under $S$ is denoted $(S, w)$ and is called the coefficient of $w$ in $S$. The set of formal power series over $A$ with coefficients in $K$ is denoted by $K\langle\langle A\rangle\rangle$. If there are only finitely many coefficients of a formal power series which are nonzero, then it is called a polynomial. The set of polynomials over $A$ with coefficients in $K$ is denoted by $K\langle A\rangle$. We can also use a standard notation for a formal power series $S \in K\langle\langle A\rangle\rangle$ by writing:

$$
S=\sum_{w \in A^{*}}(S, w) w
$$


Given two power series $S$ and $T$, we can define their sum by:

$$
(S+T, w)=(S, w)+(T, w),
$$

for each word $w \in A^{*}$. We may also define the product of $S$ and $T$ by:

$$
(S T, w)=\sum_{u v=w}(S, u)(T, v),
$$

where $u, v, w \in A^{*}$ and clearly the summation is finite for each word $w$. Two external operations of $K$ on $K\langle\langle A\rangle\rangle$ are given by:

$$
(k S, w)=k(S, w), \quad(S k, w)=(S, w) k,
$$

for each $w \in A^{*}$ where $k \in K$ (note that $K$ is not required to have commutative multiplication in general).

A formal power series, $S$, is called proper if $(S, \varepsilon)=0$, i.e., the coefficient of the empty word in $S$ is 0 . For a proper formal power series $S$, we may define the star operation:

$$
S^{*}=\sum_{i \geq 0} S^{i} .
$$

The rational operations in $K\langle\langle A\rangle\rangle$ are the sum, product, star operation and the two external products. A subset of $K\langle\langle A\rangle\rangle$ is rationally closed if it is closed under the rational operations. The smallest subset of $K\langle\langle A\rangle\rangle$ containing a subset $E$, is called the rational closure of $E$. A formal power series $S$ is called $K$-rational if it is contained within the rational closure of $K\langle A\rangle$ (the set of polynomials).

If $L$ is any language over an alphabet $A$, then its characteristic series (which we denote by $\operatorname{char}(L)$ ), is the formal power series $S \in K\langle\langle A\rangle\rangle$ :

$$
S=\operatorname{char}(L)=\sum_{w \in L} w,
$$

i.e., it is the series $S$ such that $(S, w)=1$ if $w \in L$ and 0 if $w \notin L$.

We may also define the Hadamard product of two series $S, T \in K\langle\langle A\rangle\rangle$ by:

$$
S \odot T=\sum_{w \in A^{*}}(S, w)(T, w) w .
$$

It was shown by Schützenberger that the Hadamard product of two $K$ rational formal power series is also $K$-rational [20].

Furthermore, a formal power series $S \in K\langle\langle A\rangle\rangle$ is called recognizable if there exists an integer $n \geq 1$, two vectors $\rho, \tau \in K^{n}$ and a monoid homomorphism

$$
\mu: A^{*} \mapsto K^{n \times n},
$$


such that for all words $w \in A^{*}$,

$$
(S, w)=\rho^{T} \mu(w) \tau .
$$

If such elements exist, then $(\rho, \mu, \tau)$ is called a linear representation of the formal power series $S$.

The following fundamental theorem was originally shown by Kleene for formal power series with boolean coefficients and later extended by Schützenberger to arbitrary semirings:

Theorem 1 (Schützenberger, 1961 [19]). A formal power series is rational if and only if it is recognizable.

For details of this proof, see also [6] or [18].

\section{Hilbert's Tenth Problem}

In 1900, David Hilbert presented a lecture entitled "Mathematische Probleme" in which he posed 23 open problems for the coming centuries. The tenth problem, which is the only decision problem of the list, concerns the solvability of Diophantine equations and can be stated:

Hilbert's Tenth Problem Given a Diophantine equation with any number of unknown quantities and with rational integral numerical coefficients: To devise a process according to which it can be determined by a finite number of operations whether the equation is solvable in rational integers.

The problem remained open for 70 years until a "negative solution" to the problem was shown (in other words, it was shown to be undecidable, although the notion of algorithmic unsolvability was not known at the time) in 1970 by Y. Matiyasevich building upon earlier work of many mathematicians, including M. Davis, H. Putman and J. Robinson. For more details of the history of the problem as well as the full proof of the undecidability of this theorem, see [15]. Note that we may, without loss of generality, restrict the problem to that whereby the solution is over natural numbers rather than rational integers, see $[15$, p.6].

It is well known that we may reduce Hilbert's tenth problem to a problem in formal power series, namely the problem of determining for a $\mathbb{Z}$-rational formal power series $S \in \mathbb{Z}\langle\langle A\rangle\rangle$, whether there exists any word $w \in A^{*}$ such that $(S, w)=0$. We shall now show this reduction, see also [18].

Let $P\left(n_{1}, n_{2}, \ldots, n_{k}\right)$ be an integer polynomial with $k$ variables (the variables take natural number values). We show a construction of a $\mathbb{Z}$-rational formal power series $S$ over monoid $A=\{x, y\}$ with coefficients in the natural numbers, such that for any word of the form $w=x^{n_{1}} y x^{n_{2}} y \cdots y x^{n_{k}} \in A^{*}$, where $n_{i} \geq 0$ for each $1 \leq i \leq k$, it holds that $(S, w)=P\left(n_{1}, n_{2}, \ldots, n_{k}\right)^{2}$ and for any word, $u$, not of this form, $(S, u)=1$. Thus, it follows that there 
exists some word $w \in A^{*}$ such that $(S, w)=0$ if and only if the polynomial $P$ has a solution in natural numbers. Due to the undecidability of the latter problem, the problem on formal power series is also therefore undecidable.

We shall now give the details of the construction as in [18, p.73]. For each index $1 \leq j \leq k$, define:

$R_{j}=\left(\sum_{n_{1}, \ldots, n_{j-1} \geq 0} x^{n_{1}} y x^{n_{2}} y \cdots x^{n_{j-1}} y\right)\left(\sum_{n_{j} \geq 0} n_{j} x^{n_{j}}\right)\left(\sum_{n_{j+1}, \ldots, n_{k} \geq 0} y x^{n_{j+1}} y \cdots x^{n_{k}}\right)$,

and it is not difficult to show that each series $R_{j}$ is $\mathbb{N}$-rational. Furthermore, by examining the product we see that:

$$
\left(R_{j}, x^{n_{1}} y x^{n_{2}} y \cdots y x^{n_{k}}\right)=n_{j} .
$$

It can now be seen that the series $R \in \mathbb{Z}\langle\langle A\rangle\rangle$ with the required property can be constructed using the Hadamard product, addition and subtraction of the series $R_{1}, R_{2}, \ldots, R_{k}$. However, we may note that any word $w$ not of the form $x^{n_{1}} y x^{n_{2}} y \cdots y x^{n_{k}}$ will have the property that $(R, w)=0$. Thus, we finally take the series:

$$
S=R \odot R+\operatorname{char}\left(\left(x^{*} y\right)^{k-1} x^{*}\right)^{C},
$$

where the superscript $C$ denotes the complement. This formal power series is clearly still $\mathbb{Z}$-rational and note that $R \odot R$ ensures that each element is positive or 0 . Thus $S$ contains a zero for some word if and only if that word is an encoding of a correct solution to the given Diophantine equation as required.

\section{Undecidable Matrix Equations}

We shall now show a construction which will allow us to obtain the undecidability of solving a specific type of matrix equation. As above, we shall encode a Diophantine equation within an $\mathbb{Z}$-rational formal power series, but use a different underlying monoid. We shall then convert the series to a linear representation (which is guaranteed to exist due to Theorem 1) and using this representation, we shall obtain the undecidability of determining if the matrix equation has a solution.

Theorem 2. Given integral matrices $X_{1}, X_{2}, \ldots, X_{k}$ of dimension $n \times n$, it is algorithmically undecidable to determine whether there exists a solution to the equation:

$$
X_{1}^{i_{1}} X_{2}^{i_{2}} \cdots X_{k}^{i_{k}}=Z
$$

where $Z$ denotes the zero matrix and $i_{1}, i_{2}, \ldots, i_{k} \in \mathbb{N}$ are unknowns ${ }^{1}$.

\footnotetext{
${ }^{1}$ This holds even for fixed values $n$ and $k$ depending upon the minimal degree and number of variables required in the polynomial for the proof of undecidability of Hilbert's tenth problem.
} 
Proof. Let $P\left(n_{1}, n_{2}, \ldots, n_{k}\right)$ denote a polynomial with integer coefficients and let $A=\left\{x_{1}, x_{2}, \ldots, x_{k}\right\}$ be an alphabet. Our first step shall be to construct a $\mathbb{Z}$-rational formal power series ${ }^{2}, S \in \mathbb{N}\langle\langle A\rangle\rangle$, such that for any word of the form $w=x_{1}^{n_{1}} x_{2}^{n_{2}} \cdots x_{k}^{n_{k}} \in A^{*}$, it holds that $(S, w)=P\left(n_{1}, n_{2}, \ldots, n_{k}\right)^{2}$ and for any word $u$ not of this form, we have $(S, u)=1$.

The initial construction is similar to that used in Section 3. Instead of encoding the argument of the polynomial within a binary alphabet however, we use a separate letter for each variable in the encoding. Thus, for each index $1 \leq j \leq k$, we define:

$R_{j}=\left(\sum_{n_{1}, \ldots, n_{j-1} \geq 0} x_{1}^{n_{1}} x_{2}^{n_{2}} \cdots x_{j-1}^{n_{j-1}}\right)\left(\sum_{n_{j} \geq 0} n_{j} x_{j}^{n_{j}}\right)\left(\sum_{n_{j+1}, \ldots, n_{k} \geq 0} x_{j+1}^{n_{j+1}} \cdots x_{k}^{n_{k}}\right)$,

and as previously, we see that each series $R_{j}$ is $\mathbb{N}$-rational. We now have the required property that:

$$
\left(R_{j}, x_{1}^{n_{1}} x_{2}^{n_{2}} \cdots x_{k}^{n_{k}}\right)=n_{j} .
$$

We can thus create a rational formal power series $R \in \mathbb{Z}\langle\langle A\rangle\rangle$ using the Hadamard product, addition and subtraction of the series $R_{1}, R_{2}, \ldots, R_{k}$ in a straightforward manner. To complete the encoding, we must make words not of the correct form have nonzero coefficients, thus we define the series:

$$
S=R \odot R+\operatorname{char}\left(x_{1}^{*} x_{2}^{*} \cdots x_{k}^{*}\right)^{C},
$$

where again, $\operatorname{char}(L)$ denotes the characteristic series of the language $L$ and the superscript $C$ denotes the complement of the series. Thus $S$ has the property that if any word $w \in A^{*}$ is such that $(S, w)=0$, then $w$ is of the form $x_{1}^{n_{1}} x_{2}^{n_{2}} \cdots x_{k}^{n_{k}}$ where $n_{1}, n_{2}, \ldots, n_{k} \in \mathbb{N}$, and it holds that $P\left(n_{1}, n_{2}, \ldots, n_{k}\right)=0$. Since it is undecidable if $P$ has any such solution in natural numbers, determining whether $S$ has a zero coefficient for any word $w \in A^{*}$ is undecidable.

Now, using Theorem 1 , there exists an integer $n \geq 1$, two column vectors $\rho, \tau \in \mathbb{Z}^{n}$ and a monoid morphism $\mu: A^{*} \mapsto \mathbb{Z}^{n \times n}$ such that for any word $w \in A^{*}:$

$$
(S, w)=\rho^{T} \mu(w) \tau,
$$

and $(\rho, \mu, \tau)$ is called a linear representation of the $\mathbb{Z}$-rational series $S$.

We shall not discuss how to convert between rational formal power series and linear representations, see [18] for details. Suffice it to say that in such a conversion, we may assume that $\rho$ is of the form $(1,0, \ldots, 0)^{T}$ and $\tau$ is of the form $((S, \varepsilon), 0, \ldots, 0,1)^{T}$ where $\varepsilon$ denotes the empty word and the top left element of $\mu(w)$ is 0 whenever $w \neq \varepsilon$. We can also see for a non-empty

\footnotetext{
${ }^{2}$ The series $S$ is $\mathbb{Z}$-rational but the coefficients are natural numbers.
} 
word, $w \in A^{+}$that $\rho^{T} \mu(w) \tau=\mu(w)_{1, n}$, i.e., the value $(S, w)$ is given in the top right element of $\mu(w)$.

If $\Gamma=\left\{\mu\left(x_{1}\right), \mu\left(x_{2}\right), \ldots, \mu\left(x_{k}\right)\right\} \subseteq \mathbb{Z}^{n \times n}$, then we obtain an undecidable scalar reachability problem for the semigroup generated by $\Gamma$, with the vectors $\rho, \tau$ and scalar 0 . As the final step, we shall show how to obtain the matrix equation given in the theorem.

First note that $(S, \varepsilon)$ is only present in the $\tau$ vector for the case when we have a word of 0 length, otherwise, due to the construction in [18], $(S, \varepsilon)$ will be multiplied by 0 for any non-empty word (since the left most column of $\mu(w)$ has all zero elements for all $\left.w \in A^{+}\right)$. Since we may check if $(S, \varepsilon)=0$ independently, we may ignore this value and take the vector $\tau=(0,0, \ldots, 1)^{T}$. Let us now define $X_{i}=\mu\left(x_{i}\right)$ for $1 \leq i \leq k$ and two new matrices:

$$
X_{0}=\left(\begin{array}{cccc}
1 & 0 & \cdots & 0 \\
0 & 0 & \cdots & 0 \\
\vdots & \vdots & \ddots & \vdots \\
0 & 0 & \cdots & 0
\end{array}\right), \quad X_{k+1}=\left(\begin{array}{cccc}
1 & 0 & \cdots & 0 \\
0 & 0 & \cdots & 0 \\
\vdots & \vdots & \ddots & \vdots \\
0 & 0 & \cdots & 1
\end{array}\right) \in \mathbb{Z}^{n \times n}
$$

i.e., $X_{k+1}$ has all zeros except elements $(1,1)$ and $(n, n)$ which are 1 . Note that $X_{0}$ and $X_{k+1}$ are both idempotent, thus $X_{0}^{2}=X_{0}$ and $X_{k+1}^{2}=X_{k+1}$. Consider now the equation:

$$
X_{0}^{i_{0}} X_{1}^{i_{1}} \cdots X_{k}^{i_{k}} X_{k+1}^{i_{k+1}}=Z,
$$

where $Z$ is the zero matrix of dimension $n$. Since $X_{0}$ and $X_{k+1}$ are idempotent, the powers $i_{0}$ and $i_{k+1}$ are irrelevant unless they equal 0 . If $i_{0}$ or $i_{k+1}$ equals 0 , then the corresponding matrix equals the identity matrix. We can clearly see below however that this does not affect the validity of the theorem even if either of these matrices equals the identity matrix (in fact we will get more nonzero elements).

Note that for any matrix $M \in \mathbb{Z}^{n \times n}$ :

$$
X_{0} M X_{k+1}=\left(\begin{array}{cccc}
M_{1,1} & \cdots & 0 & M_{1, n} \\
0 & \cdots & 0 & 0 \\
\vdots & \ddots & \vdots & \vdots \\
0 & \cdots & 0 & 0
\end{array}\right)
$$

and $\rho^{T} M \tau=M_{1, n}$. In the construction of [18] each matrix in the image of $\mu$ has a zero first column, thus in an equation of the form $X_{0}^{i_{0}} X_{1}^{i_{1}} \cdots X_{k}^{i_{k}} X_{k+1}^{i_{k+1}}$, the top left element equals 1 if and only if $i_{1}=i_{2}=\cdots=i_{k}=0$ (all central powers are zero corresponding to the empty word and giving the identity matrix), and we discount this case since we may check $(S, \varepsilon)$ separately as mentioned. Assume then that the top left element equals 0. Thus, Eq. (3) holds if and only if $M_{1, n}=\rho^{T} M \tau=0$, if and only if $P\left(n_{1}, n_{2}, \ldots, n_{k}\right)=0$. 
Since determining if $P\left(n_{1}, n_{2}, \ldots, n_{k}\right)=0$ is undecidable, the solvability of Eq. (3) for unknowns $i_{0}, i_{1}, \ldots, i_{k+1} \in \mathbb{N}$ is also undecidable as required.

We can note that the solvability of Eq. (3) is decidable (even in polynomial time) when all matrices are commutative, see [1].

\section{Decidable Cases}

We shall now consider decidable cases, in contrast to the results of the last section. We show that the "A-B-C Problem" is decidable in polynomial time even for non-commuting matrices $A, B, C$.

Let us state the following theorem from [1] which will be useful in this section:

Theorem 3. [1, Theorem 1.4] Let $M_{1}, M_{2}, \ldots, M_{h}, N_{1}, N_{2}, \ldots, N_{k} \subseteq \mathbb{F}^{n \times n}$ be commuting matrices (with $h, k$ fixed) and $P, Q \subseteq \mathbb{F}^{n \times l}$ (where $\mathbb{F}$ is an algebraic number field), it is decidable in polynomial time whether there exists any solutions to:

$$
\left(\prod_{i=1}^{h} M_{i}^{x_{i}}\right) P=\left(\prod_{j=1}^{k} N_{j}^{y_{j}}\right) Q,
$$

where $x_{1}, \ldots, x_{h}, y_{1}, \ldots, y_{k}$ are non-negative integers. If such a set of solutions exists, it can be found in polynomial time.

Note that from this theorem it holds that we may decide if the intersection of two commutative semigroups $S_{1}, S_{2}$ generated by $\left\{M_{1}, M_{2}, \ldots, M_{h}\right\}$ and $\left\{N_{1}, N_{2}, \ldots, N_{k}\right\}$ respectively are empty by setting $P=Q=I$ where $I$ is the identity matrix (although we need to remove the trivial solution where all exponents equal zero which is easy to do by increasing the dimension of all matrices by 1 or removing the trivial solution from those considered by Lenstra's algorithm in [1]). The same problem for non-commuting matrices was shown to be undecidable by A. Markov in 1947, [16]. The undecidability bounds were improved in the recent papers [10] and [3].

We shall now use Theorem 3 to show that the A-B-C problem is decidable for non-commutative matrices over an algebraic number field in any dimension.

Theorem 4. Given three matrices $A, B, C \in \mathbb{F}^{n \times n}$ (where $\mathbb{F}$ is an algebraic number field), it is decidable if there exists any $i, j \geq 0$ such that:

$$
A^{i} B^{j}=C .
$$

Proof. Note that the matrix product $A^{i} B^{j}=C$ can be rewritten

$$
\left(\left(B^{T}\right)^{j} \otimes A^{i}\right) \operatorname{vec}\left(I_{n}\right)=\operatorname{vec}(C)
$$


where $I_{n}$ is the $n$-dimensional identity by applying Eq. (1) from Section 2.1 .

Iteratively applying the mixed product property of Kronecker products, Eq. (2), we see that:

$$
\left(\left(B^{T}\right)^{j} \otimes A^{i}\right)=\left(\left(B^{T}\right)^{j} \otimes I\right)\left(I \otimes A^{i}\right)=\left(B^{T} \otimes I\right)^{j}(I \otimes A)^{i},
$$

and note that matrices $\left(B^{T} \otimes I\right)$ and $(I \otimes A)$ commute. Thus the problem becomes: "Does there exist an $i, j \geq 0$ such that:

$$
\left(B^{T} \otimes I\right)^{j}(I \otimes A)^{i} \operatorname{vec}\left(I_{n}\right)=\operatorname{vec}(C)
$$

is satisfied?". Since the matrices are now commutative, this is an instance of Eq. (4) where $h=2, k=1, M_{1}=\left(B^{T} \otimes I\right), M_{2}=(I \otimes A), N_{1}=I$, $P=\operatorname{vec}\left(I_{n}\right)$ and $Q=\operatorname{vec}(C)$ and by Theorem 3, this problem is decidable.

We shall now show a relation of the above result to Skolem's problem (also called Pisot's problem), which we shall soon define after some preliminary definitions. A sequence of integers $\left(u_{i}\right)_{i=0}^{\infty}$ is called a linear recurrent sequence if it satisfies the recurrence $u_{n}=u_{n-1} r_{k-1}+u_{n-2} r_{k-2}+\cdots+u_{n-k} r_{0}$, where $r_{i}$ are fixed integers called the recurrence coefficients. The first $k$ values, $u_{0}, u_{1}, \cdots, u_{k-1}$ are called the initial conditions of the sequence.

Skolem's Problem - Given the initial conditions and recurrence coefficients of a linear recurrent sequence, $\left(u_{i}\right)_{i=0}^{\infty}$, determine whether there exists an integer $i \geq 0$ such that $u_{i}=0$.

The decidability status of Skolem's problem is a long standing open problem. It was recently shown to be decidable for linear recurrences of depth 5 , see [11]. The following theorem concerning the mortality problem ${ }^{3}$ was recently proven:

Theorem 5 ([2]). Skolem's Problem with depth $k$ recurrences can be reduced to the Mortality Problem for a semigroup generated by a pair of $k$ dimensional integral matrices.

Utilizing this result and Theorem 4, we obtain the following corollary.

Corollary 6. There exist integral matrices $P, X, Z \in \mathbb{Z}^{k \times k}$ (where $Z$ is the zero matrix) such that determining if $P X^{i}=Z$ or $X^{i} P=Z$ are solvable for some $i \geq 0$, is decidable, but determining if $P X^{i} P=Z$ has a solution is equivalent to Skolem's problem.

Proof. In the proof of Theorem 5, we have two integral matrices, $P, X \in$ $\mathbb{Z}^{k \times k}$ such that $P$ has a 1 in the top left element and 0 everywhere else. It follows from the proof that we can therefore state Skolem's problem as,

\footnotetext{
${ }^{3}$ Given a matrix semigroup, determine whether the zero matrix belongs to the semigroup.
} 
"Given matrices $P, X$, does there exist an $i \geq 0$ such that $P X^{i} P=Z$ where $Z$ is the zero matrix?". This proves that the decidability of Skolem's problem can be reduced to the solvability of the equation $P X^{i} P=Z$.

For the decidable cases, note that $P$ is idempotent, thus $P^{2}=P$, from which it follows that $P X^{j}=P^{i} X^{j}$ for any $i>0$ and we know from Theorem 4 that determining if there exists any $i, j \geq 0$ such that $P^{i} X^{j}=Z$ is decidable in polynomial time (if $i=0, P^{i} X^{j}=X^{j}$ which equals $Z$ for some $j$ iff $X$ is nilpotent which is easily checked). An almost identical argument holds for the solvability of $X^{i} P=Z$, and thus the corollary holds.

\section{References}

[1] L. Babai, R. Beals, J. Cai, G. Ivanyos, E. Luks, Multiplicative Equations over Commuting Matrices, Proc. 7th ACM-SIAM Sypm. on Discrete Algorithms (SODA 1996).

[2] P. Bell, Computational Problems in Matrix Semigroups, Ph. D. Thesis, The University of Liverpool, 2007.

[3] P. Bell, A Note on the Emptiness of Semigroup Intersections, Fundamenta Informaticae, 2007, 79:1-4.

[4] P. Bell, I. Potapov, On the Membership of Invertible Diagonal and Scalar Matrices, Theoretical Computer Science, 2007, 372:37-45.

[5] D. Bernstein, Matrix Mathematics, Princeton University Press, 2005.

[6] J. Berstel, C. Reutenauer, Rational Series and Their Languages, Springer-Verlag, 1988.

[7] J. Cai, R. Lipton, Y. Zalcstein, The Complexity of the Membership Problem for 2-Generated Commutative Semigroups of Rational Matrices, Proc. of Foundations of Computer Science (FOCS 1994), 35.

[8] J. Cassaigne, T. Harju, J. Karhumäki, On the Undecidability of Freeness of Matrix Semigroups, Internat. J. Algebra Comput., 1999, 9(3-4):295305.

[9] V. Halava, T. Harju, M. Hirvensalo, Undecidability Bounds for Integer Matrices using Claus Instances, Internat. J. of Foundations of Comp. Sci., Vol. 18, Num. 5, 2007.

[10] V. Halava, T. Harju, On Markov's Undecidability Theorem for Integer Matrices, Semigroup Forum, (to appear), 2007.

[11] V. Halava, T. Harju, M. Hirvensalo, J. Karhumäki, Skolem's Problem On the Border between Decidability and Undecidability, TUCS Technical Report, Num. 683, 2005. 
[12] R. Kannan, R. Lipton, Polynomial-Time Algorithm for the Orbit Problem, Journal of ACM, Vol. 33, Num. 4, 1986, 808-821.

[13] D. Klarner, J. Birget, W. Satterfield, On the Undecidability of the Freeness of Integer Matrix Semigroups, Internat. J. Algebra Comput. 1, Num. 2, 1991, 223-226.

[14] A. Lisitsa, I. Potapov, Membership and Reachability Problems for RowMonomial Transformations, Mathematical Foundations of Computer Science (MFCS 2004), LNCS 3135, 623-634.

[15] Y. Matiyasevich, Hilbert's Tenth Problem, MIT Press, Cambridge, London, 1993.

[16] A. Markov, On Certain Insoluable Problems Concerning Matrices, Doklady Akad. Nauk SSSR, 1947, 57:539-542.

[17] M. Paterson, Unsolvability in 3x3 Matrices, Studies in Applied Mathematics, 1970, 49:105-107.

[18] A. Salomaa, M. Soittola, Automata-Theoretic Aspects of Formal Power Series, Springer-Verlag, 1978.

[19] M. P. Schützenberger, On the Definition of a Family of Automata, Information and Control, 1961, 4:245-270.

[20] M. P. Schützenberger, On a Theorem of R. Jungen, Proc. Amer. Math. Soc., 13:885-890, ISSN 0002-9939, 1962. 